\title{
Spontaneous emission of an atom near a wedge
}

\author{
F.S.S. Rosa \\ Theoretical Division, Los Alamos National Laboratory, Los Alamos, NM 87545, USA \\ T.N.C. Mendes \\ Universidade Federal Fluminense - Niterói, RJ, 24210-340, Brazil \\ A. Tenório \\ Centro Brasileiro de Pesquisas Físicas - Rio de Janeiro, RJ, 22290-180, Brazil \\ C. Farina \\ Universidade Federal do Rio de Janeiro - Rio de Janeiro, RJ, 21941-972 , Brazil
}

\begin{abstract}
It is a well known fact that the presence of material bodies in the vicinity of an atom affects its interaction with the always present quantized electromagnetic field. In this paper, we focus on how the spontaneous emission rate of a given excited atom is altered when this atom is placed inside a perfectly conducting wedge. We begin by briefly presenting the formalism on which our calculations are founded, proceeding then to a long but straightforward calculation of the transition rate. We present results for a general atom but, for the sake of simplicity, we narrow them down to an effective two-level system in our numerical investigations. The oscillatory pattern for the spontaneous emission rate of the atom as we vary its relative position to the wedge as well as the phenomenon of suppression are shown in a couple of graphs.

PACS numbers: 12.20.Ds, 34.20.Cf
\end{abstract}

\section{INTRODUCTION}

Last year we have celebrated the ninetieth anniversary of A. Einstein's historical paper on his theory of radiation [1, the final paper of a series that began more than a decade earlier with his 1905 work on the quantization of the radiation field. By trying to explain how atoms could be in thermal equilibrium with the radiation field, he introduced the novel concepts of spontaneous and stimulated emission of radiation [2, that again outlined how unusual quantum mechanics could be and reheated a dormant discussion about causality in the quantum scale 3. However, despite its huge achievements this remarkable work had at least one shortcoming, since it did not furnish the means for evaluation of the spontaneous emission rate. This had to wait another ten years, until P.A.M. Dirac successfully calculated it in a paper that most regard as the birth of quantum electrodynamics [5].

Once established the framework of the modern quantum field theory, a meaningful question to ask is how spontaneous emission (SE) was affected by the presence of boundaries. That nontrivial boundary conditions could modify the SE rate is very clear from the QED standpoint, since it is the quantized electromagnetic field that causes SE and the field is definitely affected by boundary conditions [6]. This issue was firstly considered by E.M. Purcell 7 in the forties, and subsequent experimental work showed that SE could be strongly modified if boundaries were present [8, 9]. The first explicit theoretical results [10, 11] for parallel conducing plates also supported the conclusion that boundary conditions could significantly enhance or suppress the SE rate 12 .

In this work we carry on the analysis on how boundaries may influence the SE process. We investigate the behavior of an atom inside a wedge made of conducting plates. The reason why we chose such a setup is twofold. Firstly, the wedge configuration is a relatively simple system that allows the study of nontrivial curvature effects in the zero-point field [13 16, due to the presence of the cusp. In addition, the wedge geometry has already been used in experiments [17-although thereabouts the wedge aspect was not important, it may probably be in the future. Such configuration may also be relevant for trapping atoms in excited states, since a suited geometry could lead to locations where the potential well disturbing the atom is deep and the SE rate is small.

The plan of this paper is arranged as follows. In the next section we present the necessary formalism in some detail, and show how it leads to a systematic way of evaluating SE rates. In section III we define our problem and proceed with the calculations needed in order to bring the result to the most convenient form for numerical investigations, leaving the fourth section to conclusions and perspectives.

\section{TRANSITION RATES OF A SYSTEM COUPLED TO A RESERVOIR}

\section{A. General formalism}

Let be a small system $\mathcal{S}$ characterized by a time dependent density matrix operator $\rho_{S}(t)$ which interacts with a large system $\mathcal{R}$ which can be treated as a reser- 
voir and characterized by a time independent density matrix operator $\rho_{R}$. The Hamiltonian of the whole system $\mathcal{S}+\mathcal{R}$ is $H=H_{S}+H_{R}+V$, where $H_{S}\left(H_{R}\right)$ is the unperturbed Hamiltonian of $\mathcal{S}(\mathcal{R})$ with eigenstates $|a\rangle(|\mu\rangle)$ and with eigenvalues $E_{a}\left(E_{\mu}\right)$. By hypothesis, the interaction hamiltonian between $\mathcal{S}$ and $\mathcal{R}$ is

$$
V=\sum_{j} S_{j} R_{j}
$$

where $S_{j}$ and $R_{j}$ are compatible observables associated to $\mathcal{S}$ and $\mathcal{R}$ respectively. Assuming that correlations occurring between $\mathcal{S}$ and $\mathcal{R}$ last a time $\tau_{c}$ and that variations of the observables in $\mathcal{S}$ are characterized by a time scale $T_{S}$, one is able to write a coarse grained rate equation for the time evolution of the diagonal elements of $\rho_{S}(t)$ in the base ket $|\{a\}\rangle[18,19$,

$$
\frac{\mathrm{d}}{\mathrm{d} t} \rho_{a a}^{S}(t)=\sum_{c}\left(\rho_{c c}^{S}(t) \Gamma_{c \rightarrow a}-\rho_{a a}^{S}(t) \Gamma_{a \rightarrow c}\right)
$$

where $d t$ is not infinitesimal but satisfies the inequality $\tau_{c} \ll d t \ll T_{S}$, provided of course that such a relation holds for some $d t$. The factor $\Gamma_{c \rightarrow a}$ is defined by

$$
\begin{array}{r}
\Gamma_{c \rightarrow a}=\frac{2 \pi}{\hbar} \sum_{\mu} p_{\mu} \sum_{\nu}|\langle\mu, c|V| \nu, a\rangle|^{2} . \\
\delta\left(E_{\mu}+E_{c}-E_{\nu}-E_{a}\right)
\end{array}
$$

where $\rho_{a b}^{S}=\left\langle a\left|\rho_{S}\right| b\right\rangle$ and $p_{\mu}$ is the statistical weight for a state $|\mu\rangle$ of the reservoir. We see that $\Gamma_{c \rightarrow a}$ may be interpreted as the probability (per unit time) of a transition in the system between the states $|c\rangle$ and $|a\rangle$. Equation (3) is precisely the Fermi's golden rule.

In order to obtain the exchange energy rates, one must consider the time derivative of the average value of the hamiltonian of the system. For a given eigenstate $|a\rangle$ of the system, a simple application of Eq. (2) shows that the net exchange energy rate between $\mathcal{S}$ and $\mathcal{R}$ is

$$
\frac{\mathrm{d}\left\langle H_{S}\right\rangle_{a}}{\mathrm{~d} t}=\dot{\rho}_{a a}^{S} E_{a}=-\sum_{b} \hbar \omega_{a b} \Gamma_{a \rightarrow b}
$$

where $\omega_{a b}=\left(E_{a}-E_{b}\right) / \hbar$. Following the work developed by J. Dalibard et al. [18, 19], we now proceed to recast the previous result into a more intuitive form that clearly exhibits the roles played by $\mathcal{S}$ and $\mathcal{R}$. Let us begin by defining the symmetric correlation function $C_{j k}^{R}(\omega)$ and the linear susceptibility $\chi_{j k}^{R}(\omega)$ of the reservoir in the frequency space [20, 21],

$$
\begin{aligned}
\hat{C}_{j k}^{R}(\omega)= & \pi \sum_{\mu} p_{\mu} \cdot \\
& \sum_{\nu} R_{\mu \nu}^{j} R_{\nu \mu}^{k}\left[\delta\left(\omega+\omega_{\mu \nu}\right)+\delta\left(\omega-\omega_{\mu \nu}\right)\right], \\
\hat{\chi}_{j k}^{R}(\omega)= & \hat{\chi}_{j k}^{\prime R}(\omega)+\imath \hat{\chi}_{j k}^{\prime \prime R}(\omega) \\
\hat{\chi}_{j k}^{\prime R}(\omega)= & -\frac{1}{\hbar} \sum_{\mu} p_{\mu} \cdot \\
& \sum_{\nu} R_{\mu \nu}^{j} R_{\nu \mu}^{k}\left[\mathcal{P} \frac{1}{\omega_{\mu \nu}+\omega}+\mathcal{P} \frac{1}{\omega_{\mu \nu}-\omega}\right], \\
\hat{\chi}_{j k}^{\prime \prime R}(\omega)= & \frac{\pi}{\hbar} \sum_{\mu} p_{\mu} \cdot \\
& \sum_{\nu} R_{\mu \nu}^{j} R_{\nu \mu}^{k}\left[\delta\left(\omega_{\mu \nu}+\omega\right)-\delta\left(\omega_{\mu \nu}-\omega\right)\right],
\end{aligned}
$$

where $R_{\mu \nu}^{j}=\left\langle\mu\left|R_{j}\right| \nu\right\rangle, \omega_{\mu \nu}=\left(E_{\mu}-E_{\nu}\right) / \hbar$ and $\mathcal{P}$ is the principal part. The equivalent expressions for the system $\mathcal{S}$ are

$$
\begin{aligned}
\hat{C}_{j k}^{S, a}(\omega) & =\pi \sum_{n} S_{a n}^{j} S_{n a}^{k}\left[\delta\left(\omega+\omega_{a n}\right)+\delta\left(\omega-\omega_{a n}\right)\right] \\
\hat{\chi}_{j k}^{\prime S, a}(\omega) & =-\frac{1}{\hbar} \sum_{n} S_{a n}^{j} S_{n a}^{k}\left[\mathcal{P} \frac{1}{\omega_{a n}+\omega}+\mathcal{P} \frac{1}{\omega_{a n}-\omega}\right] \\
\hat{\chi}_{j k}^{\prime \prime S, a}(\omega) & =\frac{\pi}{\hbar} \sum_{n} S_{a n}^{j} S_{n a}^{k}\left[\delta\left(\omega_{a n}+\omega\right)-\delta\left(\omega_{a n}-\omega\right)\right]
\end{aligned}
$$

with $S_{a b}^{j}=\left\langle a\left|S_{j}\right| b\right\rangle$. It is now a matter of straightforward algebra to show that (4) may be rewritten in terms of these new quantities

$$
\begin{aligned}
\frac{\mathrm{d}\left\langle H_{S}\right\rangle_{a}}{\mathrm{~d} t} & =\dot{\mathcal{Q}}_{a}=\dot{\mathcal{Q}}_{a}^{f r}+\dot{\mathcal{Q}}_{a}^{r r} \\
\dot{\mathcal{Q}}_{a}^{f r} & =\sum_{j, k} \int_{-\infty}^{\infty} \frac{\mathrm{d} \omega}{2 \pi} \omega \hat{\chi}_{j k}^{\prime \prime S, a}(\omega) \hat{C}_{k j}^{R}(\omega) \\
\dot{\mathcal{Q}}_{a}^{r r} & =-\sum_{j, k} \int_{-\infty}^{\infty} \frac{\mathrm{d} \omega}{2 \pi} \omega \hat{\chi}_{j k}^{\prime \prime R}(\omega) \hat{C}_{k j}^{S, a}(\omega)
\end{aligned}
$$

The physical interpretations of $\dot{\mathcal{Q}}_{a}^{f r}$ and $\dot{\mathcal{Q}}_{a}^{r r}$ are simple. The former may be understood as the power kicked into the system $\mathcal{S}$ by the fluctuations of the reservoir $(f r)$, while the latter represents the power lost to the reservoir due to fluctuations of the system itself. This last term is also called the reservoir reaction contribution $(\mathrm{rr})$ since these fluctuations of the system affect the reservoir, which then back reacts on the system [18, 19].

\section{B. Dipole interacting with the radiation field}

Let us now take our system to be a small neutral entity, characterized by the set of transition frequencies $\omega_{b a}$, and let us assume that it is in contact with the quantized radiation field, taken as the reservoir. The radiation eletric field may be written as

$$
\mathbf{E}(\mathbf{x}, t)=\sum_{\mathbf{k} \lambda}\left(\mathbf{f}_{\mathbf{k} \lambda}(\mathbf{x}) \mathrm{e}^{\imath \omega_{k} t} a_{\mathbf{k} \lambda}^{\dagger}+\mathbf{f}_{\mathbf{k} \lambda}^{*}(\mathbf{x}) \mathrm{e}^{-\imath \omega_{k} t} a_{\mathbf{k} \lambda}\right)
$$


where each mode is specified by a wavevector $\mathbf{k}$ and polarization $\lambda$, with $\omega_{k}=c|\mathbf{k}|$. The annihilation and creation operators $a_{\mathbf{k} \lambda}$ and $a_{\mathbf{k} \lambda}^{\dagger}$ satisfy the usual commutation relations

$$
\begin{array}{r}
{\left[a_{\mathbf{k} \lambda}, a_{\mathbf{k}^{\prime} \lambda^{\prime}}\right]=\left[a_{\mathbf{k} \lambda}^{\dagger}, a_{\mathbf{k}^{\prime} \lambda^{\prime}}^{\dagger}\right]=0,} \\
{\left[a_{\mathbf{k} \lambda}, a_{\mathbf{k}^{\prime} \lambda^{\prime}}^{\dagger}\right]=\delta_{\mathbf{k k}^{\prime}} \delta_{\lambda \lambda^{\prime}},}
\end{array}
$$

and the function $\mathbf{f}_{\mathrm{k} \lambda}(\mathbf{x})$ is the position dependent part of the mode $\mathbf{k} \lambda$ and carry the information about the boundary conditions and possible source contributions.

Let us assume that the dimensions of the system $\mathcal{S}$ are too small compared to any of its transition wavelengths, that is, $a_{0} \ll 2 \pi c / \omega_{a b}$, where $a_{0}$ is the largest dimension of the system, and also that $\mathcal{S}$ is non-relativistic. Then the interaction part of the hamiltonian may be approximated by the dipole interaction of a particle and the electric field

$$
\begin{aligned}
V(\mathbf{x}, t) & =-\mathbf{d} \cdot \mathbf{E}(\mathbf{x}, t)= \\
& -\sum_{\mathbf{k} \lambda} \sum_{j}\left(d_{j} f_{\mathbf{k} \lambda}^{j}(\mathbf{x}) \mathrm{e}^{\imath \omega_{k} t} a_{\mathbf{k} \lambda}^{\dagger}+\text { h.c. }\right)
\end{aligned}
$$

where $\mathbf{d}$ is the dipole moment operator of the system $f_{\mathbf{k} \lambda}^{j}(\mathbf{x})$ is the $j$-component of $\mathbf{f}_{\mathbf{k} \lambda}(\mathbf{x})$ and the index $j=1,2,3$ runs over the directional unitary vectors of a given tridimensional coordinate system. Assuming that the susceptibility and the correlation function of $\mathcal{S}$ are diagonal in the appropriate coordinate system, we have

$$
\begin{aligned}
& \hat{C}_{j k}^{S, a}(\omega) \equiv \alpha_{a j}^{(-)}(\omega) \\
& =-\pi \hbar \sum_{b} \frac{\alpha_{a b}^{j} \omega_{a b}}{2}\left[\delta\left(\omega_{a b}+\omega\right)+\delta\left(\omega_{a b}-\omega\right)\right] \\
& \hat{\chi}_{j k}^{\prime \prime S, a}(\omega) \equiv \alpha_{a j}^{(+)}(\omega) \\
& =\pi \sum_{b} \frac{\alpha_{a b}^{j} \omega_{a b}}{2}\left[\delta\left(\omega_{a b}-\omega\right)-\delta\left(\omega_{a b}+\omega\right)\right]
\end{aligned}
$$

where $\alpha_{a b}^{j}=-2\left|\left\langle a\left|d_{j}\right| b\right\rangle\right|^{2} / \hbar \omega_{a b}$.

Using last equations in (13) and (14), making $|\mu\rangle \equiv$ $\left|n_{\mathbf{k} \lambda}\right\rangle$ in equation (3) (which means a Fock state with $n$ photons in the mode $\mathbf{k} \lambda$ ) and performing the integration on $\omega$, we obtain, after some calculations,

$$
\begin{gathered}
\dot{\mathcal{Q}}_{a}^{r r}=-\sum_{j} \sum_{\mathbf{k} \lambda} c k \alpha_{a j}^{(-)}(k)\left|f_{\mathbf{k} \lambda}^{j}(\mathbf{x})\right|^{2}, \\
\dot{\mathcal{Q}}_{a}^{f r}=\sum_{j} \sum_{\mathbf{k} \lambda} c k \alpha_{a j}^{(+)}(k)\left|f_{\mathbf{k} \lambda}^{j}(\mathbf{x})\right|^{2}\left(2\left\langle n_{\mathbf{k} \lambda}\right\rangle+1\right),
\end{gathered}
$$

where $\left\langle n_{\mathbf{k} \lambda}\right\rangle$ is the average number of photons in the mode $\mathbf{k} \lambda$.

As pointed out before, equation (21) gives the contribution arising from the radiation reaction to the energy rates of the system, while equation 22 gives the corresponding contribution coming from the field fluctuations.
From 21) and 19), it is possible to see that the $(r r)$ contribution is never positive, which means that it always accounts for power that is emitted by the system. Since it also does not depend on $\left\langle n_{\mathbf{k} \lambda}\right\rangle$, we conclude that the $(r r)$ contribution comes from spontaneous processes only. On the other hand, Equations $(22)$ and $(20)$ show that the $(f r)$ contribution can have both signs, meaning that $\dot{\mathcal{Q}}_{a}^{f r}$ could represent either an emitted or an absorbed power. In addition, its dependence on $\left\langle n_{\mathbf{k} \lambda}\right\rangle$ signals that stimulated processes also play a role in the $f r$ contribution. For the special case where $\left\langle n_{\mathbf{k} \lambda}\right\rangle=0$, there is no absorbed power and the total (spontaneous) radiated power by the system is

$$
\begin{aligned}
\dot{\mathcal{Q}}_{a} & =\sum_{b, j} \dot{\mathcal{Q}}_{a b, j} \\
& =\pi c \sum_{j} \sum_{b<a} \alpha_{a b}^{j} k_{a b} \sum_{\mathbf{k} \lambda} k\left|f_{\mathbf{k} \lambda}^{j}(\mathbf{x})\right|^{2} \delta\left(k-k_{a b}\right),
\end{aligned}
$$

where $k_{a b}=\omega_{a b} / c$ and. Let us also note that the emitted power at a given permitted transition, $\dot{\mathcal{Q}}_{a b, j}$, is proporcional to the spontaneous emission rate $\Gamma_{a \rightarrow b}^{j}$ characteristic of this transition.

\section{THE SPONTANEOUS EMISSION OF AN ATOM INSIDE A WEDGE}

As far as we know, the method described in the previous section was firstly applied in atom-surface problems by D. Meschede et al. [22], where they found the level shifts and the radiation rates of an atom near a single wall. A few years later, some of the present authors revived this method to evaluate thermal corrections to the same problem [23, and also to calculate the van der Waals interaction between an atom and a perfectly conducting wedge 24]. In this paper, we intend to continue this work by evaluating the spontaneous emission rates for the atom-wedge system.

Let us consider the system described schematically in figure 1. It consists of an atom placed inside of a perfectly conducting wedge, characterized by a radius $\mathrm{R}$ and an angle $\alpha$. The location of the atom is given by its distance $\rho$ to the wedge axis and its declination $\varphi$ relative to the bisector plane, related to the auxiliary angle $\phi$ by $\varphi=\phi-\alpha / 2$. Let us further assume that this atom is characterized by a dipole moment $\mathbf{d}=-e \mathbf{r}_{e}$, where $e$ is the fundamental charge and $\mathbf{r}_{e}$ is the electron position with respect to the center of the atom. It is then clear that taking the atom as our system $\mathcal{S}$ and the electromagnetic (EM) field constrained by the presence of the wedge as the reservoir $\mathcal{R}$, we may straightforwardly apply the formalism discussed in the previous section.

According to 21)- 222, we need the modes of the EM field inside the wedge in order to evaluate the spontaneous emission rate. As they have been already obtained, 


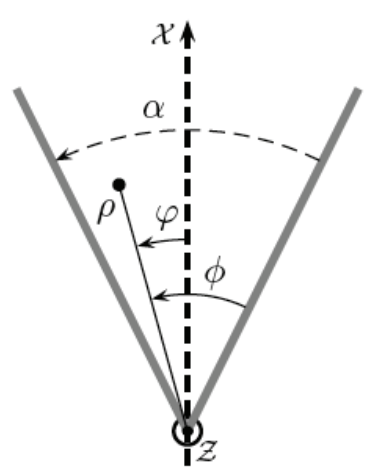

FIG. 1: The Atom-wedge system. The atom is represented by the black circle.

for instance, in [13, we will merely quote them

$$
\begin{gathered}
\mathbf{f}_{\mathbf{k}, m, n}^{\mathrm{TM}}(\mathbf{x})=\beta_{q m}\left(\gamma_{m, n} R\right)\left(\gamma_{m, n}^{2} \hat{z}-\imath k_{z} \nabla_{t}\right) . \\
{\left[J_{q m}\left(\gamma_{m, n} \rho\right) \sin (q m \phi) e^{-\imath k_{z} z}\right],} \\
\mathbf{f}_{\mathbf{k}, m, n}^{\mathrm{TE}}(\mathbf{x})=\imath k \beta_{q m}\left(\eta_{m, n} R\right) \cdot \\
\hat{z} \times \nabla_{t}\left[J_{q m}\left(\eta_{m, n} \rho\right) \cos (q m \phi) e^{-\imath k_{z} z}\right],
\end{gathered}
$$

where $q=\pi / \alpha, m \in \mathbb{N}^{*}$ in $24, m \in \mathbb{N}$ in 25 and

$$
\omega_{k}^{2}=c^{2}\left[\gamma_{m, n}^{2}+k_{z}^{2}\right] .
$$

The quantities $\gamma_{m, n}$ and $\eta_{m, n}$ are defined by

$$
J_{q m}\left(\gamma_{m, n} R\right)=J_{q m}^{\prime}\left(\eta_{m, n} R\right)=0 \quad n \in \mathbb{N}
$$

and

$$
\begin{aligned}
& \beta_{q m}^{2}(x)=\frac{4 q \hbar c}{\pi k} X_{q m}(x) \text { if } m \neq 0 \\
& \beta_{q m}^{2}(x)=\frac{2 q \hbar c}{\pi k} X_{q m}(x) \text { if } m=0 \\
& X_{\nu}(x)=\left[J_{\nu}^{\prime 2}(x)+\left(1-\nu^{2} / x^{2}\right) J_{\nu}^{2}(x)\right]^{-1} \\
& \nabla_{t}=\hat{\rho} \partial_{\rho}+\rho^{-1} \hat{\phi} \partial_{\phi} \\
& k^{2}=\kappa_{m n \lambda}^{2}+k_{z}^{2}
\end{aligned}
$$

with $\kappa_{m n 0}=\gamma_{m, n}$ and $\kappa_{m n 1}=\eta_{m, n}$. Inserting (24)-25 into 23 and setting $\left\langle n_{k \lambda}\right\rangle=0$, since we are at zero temperature, we get after a little manipulation

$$
\dot{\mathcal{Q}}_{a}=2 \hbar \pi c^{2} \sum_{\lambda, j} \sum_{b<a} \alpha_{a b}^{j} k_{a b} \int_{-\infty}^{\infty} \mathrm{d} k_{z} \sum_{m=-\infty}^{\infty} \sum_{n=1}^{\infty} \kappa_{|m| n \lambda}^{4} \delta\left(k_{a b}-\sqrt{\kappa_{|m| n \lambda}^{2}+k_{z}^{2}}\right) X_{q|m|}\left(\kappa_{|m| n \lambda} R\right) Q_{q|m|, n}^{j, \lambda}(\rho, \phi)
$$

where $\lambda=0,1, j=r, \phi, z$, and

$$
\begin{aligned}
Q_{q m}^{\rho, 0}\left(\kappa_{m n 0}, \rho, \phi\right) & =\frac{k_{z}^{2}}{\kappa_{m n 0}^{2}} J_{q m}^{\prime 2}\left(\kappa_{m n 0} \rho\right) \sin ^{2}(q m \phi), \\
Q_{q m}^{\phi, 0}\left(\kappa_{m n 0}, \rho, \phi\right) & =\frac{k_{z}^{2} q^{2} m^{2}}{\kappa_{m n 0}^{4} \rho^{2}} J_{q m}^{2}\left(\kappa_{m n 0} \rho\right) \cos ^{2}(q m \phi), \\
Q_{q m}^{z, 0}\left(\kappa_{m n 0}, \rho, \phi\right) & =J_{q m}^{2}\left(\kappa_{m n 0} \rho\right) \sin ^{2}(q m \phi), \\
Q_{q m}^{\rho, 1}\left(\kappa_{m n 1}, \rho, \phi\right) & =\left(1+\frac{k_{z}^{2}}{\kappa_{m n 1}^{2}}\right) \frac{q^{2} m^{2}}{\kappa_{m n 1}^{2} \rho^{2}} J_{q m}^{2}\left(\kappa_{m n 1} \rho\right) \sin ^{2}(q m \phi), \\
Q_{q m}^{\phi, 1}\left(\kappa_{m n 1}, \rho, \phi\right) & =\left(1+\frac{k_{z}^{2}}{\kappa_{m n 1}^{2}}\right) J_{q m}^{\prime 2}\left(\kappa_{m n 1} \rho\right) \cos ^{2}(q m \phi), \\
Q_{q m}^{z, 1}\left(\kappa_{m n 1}, \rho, \phi\right) & =0 .
\end{aligned}
$$

We may now use the generalized Abel-Plana formula [13, 25, which is

$$
\begin{aligned}
\sum_{n=1}^{\infty}\left(\kappa_{m n \lambda} R\right) X_{q m}\left(\kappa_{m n \lambda} R\right) f\left(\kappa_{m n \lambda} R\right)= & \frac{1}{2} \int_{0}^{\infty} d x f(x)+\frac{\pi}{4} \operatorname{Res}\left[f(z) \frac{Y_{q m}^{(\lambda)}(z)}{J_{q m}^{(\lambda)}(z)}\right]_{z=0} \\
& -\frac{1}{2 \pi} \int_{0}^{\infty} d x \frac{K_{q m}^{(\lambda)}(x)}{I_{q m}^{(\lambda)}(x)}\left[e^{-q m \pi i} f\left(e^{i \pi / 2} x\right)+e^{q m \pi i} f\left(e^{-i \pi / 2} x\right)\right]
\end{aligned}
$$

where the superscript $(\lambda)$ in the Bessel functions mean their $\lambda$-th derivative, in order to recast the expression 
(33) into

$$
\dot{\mathcal{Q}}_{a}=\hbar \pi c^{2} \sum_{\lambda, j, b} \alpha_{a b}^{j} k_{a b} \int_{-\infty}^{\infty} \mathrm{d} k_{z} \sum_{m=-\infty}^{\infty} \int_{0}^{\infty} d y y^{3} \delta\left(k_{a b}-\sqrt{y^{2}+k_{z}^{2}}\right) Q_{q|m|}^{j, \lambda}(y, \rho, \phi)+\mathcal{F}(R, \rho, \phi),
$$

where we were able to isolate all the R-dependence of $\dot{\mathcal{Q}}_{a}$ into an involved but given function $\mathcal{F}(R, \rho, \phi)$. As we are interested in the wedge without the external cap, we must take the $R \rightarrow \infty$ limit, and here is where the rearrangement we made in (41) shows its quality: it may be shown that $\mathcal{F}(R \rightarrow \infty, \rho, \phi) \rightarrow 0$ [13], leaving us only with the first term.

Although the generalized Abel-Plana formula made things simpler, expression (41) still is not very efficient for numerical investigations. However, if we rewrite the opening angle as $\alpha=\pi / q$ and restrict ourselves to integer values of the parameter $q$, it turns out that further simplification is possible. In this case we can switch $|m|$ to $m$ in (41) and carry out the $m$-summation by using an addition theorem relating Bessel functions 26.

$$
\sum_{m=-\infty}^{\infty} J_{q m}(\kappa \rho) Z_{\nu+q m}(\kappa \rho) \mathrm{e}^{2 \imath q m \phi}=\frac{1}{q} \sum_{l=0}^{q-1}(-1)^{\nu / 2} \mathrm{e}^{-\imath \nu\left(\phi+\frac{\pi l}{q}\right)} Z_{\nu}\left(2 \kappa \rho \sin \left(\phi+\frac{\pi l}{q}\right)\right)
$$

where $Z_{\nu}$ is a given solution of Bessel equation. As slightly different cases of this theorem will apply to each polarization, it is convenient to split $\dot{\mathcal{Q}}_{a}$ in its $\rho, \phi$ and $z$ contributions

$$
\dot{\mathcal{Q}}_{a}=\sum_{j} \dot{\mathcal{Q}}_{a, j}=\dot{\mathcal{Q}}_{a, \rho}+\dot{\mathcal{Q}}_{a, \phi}+\dot{\mathcal{Q}}_{a, z}
$$

$$
\begin{aligned}
\dot{\mathcal{Q}}_{a, z}=\frac{\hbar \pi c^{2}}{2 q} \sum_{b<a} \alpha_{a b}^{z} k_{a b} \int_{-\infty}^{\infty} & d k_{z} \int_{0}^{\infty} d y y^{3} \delta\left(k_{a b}-\sqrt{y^{2}+k_{z}^{2}}\right) \\
& \sum_{l=0}^{q-1}\left[J_{0}\left(2 y \rho \sin \left(\frac{\pi l}{q}\right)\right)-J_{0}\left(2 y \rho \sin \left(\phi+\frac{\pi l}{q}\right)\right)\right] .
\end{aligned}
$$

By performing the change of variables $y=k \sin \theta, k_{z}=k \cos \theta$ and using the identity [27]

$$
\int_{0}^{\pi / 2} J_{\mu}(a \sin \theta)(\sin \theta)^{\mu+1}(\cos \theta)^{2 \nu+1} \mathrm{~d} \theta=2^{\nu} \Gamma(\nu+1) a^{-\nu-1} J_{\nu+\mu+1}(a),
$$

valid when $\operatorname{Re}[\nu]>-1, \operatorname{Re}[\mu]>-1$, we may perform the trivial $k$-integration (due to the $\delta$-function) to finally put (44) in the form

$$
\dot{\mathcal{Q}}_{a, z}=-\frac{1}{2} \sum_{b<a} \hbar c\left|k_{a b}\right| \Gamma_{a \rightarrow b}^{z}\left\{\frac{2}{3}-G_{\|}\left(2\left|k_{a b}\right| \rho \sin \phi\right)-\sum_{l=1}^{q-1}\left[G_{\|}\left(2\left|k_{a b}\right| \rho \sin (\phi+\pi l / q)\right)-G_{\|}\left(2\left|k_{a b}\right| \rho \sin (\pi l / q)\right)\right]\right\},
$$

where

$$
G_{\|}(x)=\frac{\sin x}{x}+\frac{\cos x}{x^{2}}-\frac{\sin x}{x^{3}}
$$


$J_{1 / 2}(x)$ and $J_{3 / 2}(x)$. We have also defined

$$
\Gamma_{a \rightarrow b}^{j}=\frac{4}{\hbar}\left|\left\langle a\left|d_{j}\right| b\right\rangle\right|^{2}\left|k_{a b}\right|^{3},
$$

which is nothing but the spontaneous emission rate between levels a and $\mathrm{b}$ for the $j$-polarization in free space, assuming of course that $a>b$.

The calculations for $\dot{\mathcal{Q}}_{a, \rho}$ and $\dot{\mathcal{Q}}_{a, \phi}$ are a little bit more involved but rather analogous to the one we just showed.

In these cases the m-summations that we need to evaluate are

$$
\begin{array}{r}
\sum_{m=-\infty}^{\infty} m^{2} J_{q m}^{2}(\kappa \rho)\left\{\begin{array}{l}
\cos ^{2}(q m \phi) \\
\sin ^{2}(q m \phi)
\end{array}\right\}, \\
\sum_{m=-\infty}^{\infty} J_{q m}^{\prime 2}(\kappa \rho)\left\{\begin{array}{l}
\cos ^{2}(q m \phi) \\
\sin ^{2}(q m \phi)
\end{array}\right\} .
\end{array}
$$

The first ones, shown in (49), may be carried out by considering the particular case of 42 in which $Z_{\nu+q m}(\kappa \rho)=$ $J_{q m}(\kappa \rho)$ and differentiating it twice with respect to the variable $\phi$. After some algebraic manipulations we get

$$
\frac{q^{2}}{\kappa^{2} \rho^{2}} \sum_{m=-\infty}^{\infty} m^{2} J_{q m}^{2}(\kappa \rho) \cos (2 q m \phi)=\frac{1}{q} \sum_{l=0}^{q-1}\left[J_{0}\left(2 \kappa \rho \sin \psi_{l}\right) \cos ^{2} \psi_{l}-\frac{J_{1}\left(2 \kappa \rho \sin \psi_{l}\right)}{2 \kappa \rho \sin \psi_{l}}\right],
$$

where $\psi_{l}=\phi+\pi l / q$, which immediately leads to

$$
\begin{gathered}
\sum_{m=-\infty}^{\infty} m^{2} J_{q m}^{2}(\kappa \rho) \sin ^{2}(q m \phi)=\frac{1}{2 q} \sum_{l=0}^{q-1}\left[-J_{0}\left(2 \kappa \rho \sin \psi_{l}\right) \cos ^{2} \psi_{l}+J_{0}(2 \kappa \rho \sin \pi l / q) \cos ^{2} \pi l / q\right. \\
\left.+\frac{J_{1}\left(2 \kappa \rho \sin \psi_{l}\right)}{2 \kappa \rho \sin \psi_{l}}-\frac{J_{1}(2 \kappa \rho \sin \pi l / q)}{2 \kappa \rho \sin \pi l / q}\right], \\
\sum_{m=-\infty}^{\infty} m^{2} J_{q m}^{2}(\kappa \rho) \cos ^{2}(q m \phi)=\frac{1}{2 q} \sum_{l=0}^{q-1}\left[J_{0}\left(2 \kappa \rho \sin \psi_{l}\right) \cos ^{2} \psi_{l}+J_{0}(2 \kappa \rho \sin \pi l / q) \cos ^{2} \pi l / q\right. \\
\left.-\frac{J_{1}\left(2 \kappa \rho \sin \psi_{l}\right)}{2 \kappa \rho \sin \psi_{l}}-\frac{J_{1}(2 \kappa \rho \sin \pi l / q)}{2 \kappa \rho \sin \pi l / q}\right] .
\end{gathered}
$$

The remaining summations in 50 may be evaluated by making use of the identity

$$
\begin{aligned}
J_{q m}^{\prime 2}(\kappa \rho) & =\frac{q m}{\kappa \rho} J_{q m}^{\prime}(\kappa \rho) J_{q m}(\kappa \rho)-\partial_{\kappa \rho}\left[J_{q m}(\kappa \rho) J_{q m+1}(\kappa \rho)\right] \\
& +J_{q m}^{2}(\kappa \rho)-\frac{q m+1}{\kappa \rho} J_{q m}(\kappa \rho) J_{q m+1}(\kappa \rho),
\end{aligned}
$$

plus the particular cases of 42 for $Z_{\nu+q m}(\kappa \rho)=J_{q m}(\kappa \rho), J_{q m+1}(\kappa \rho)$ and its derivatives with respect to variables $\rho$ and $\phi$. This yields

$$
\begin{aligned}
& \sum_{m=-\infty}^{\infty} J_{q m}^{\prime 2}(\kappa \rho) \sin ^{2}(q m \phi)=\frac{1}{2 q} \sum_{l=0}^{q-1} {\left[J_{0}\left(2 \kappa \rho \sin \psi_{l}\right) \sin ^{2} \psi_{l}-J_{0}(2 \kappa \rho \sin \pi l / q) \sin ^{2} \pi l / q\right.} \\
&\left.-\frac{J_{1}\left(2 \kappa \rho \sin \psi_{l}\right)}{2 \kappa \rho \sin \psi_{l}}+\frac{J_{1}(2 \kappa \rho \sin \pi l / q)}{2 \kappa \rho \sin \pi l / q}\right], \\
& \sum_{m=-\infty}^{\infty} J_{q m}^{\prime 2}(\kappa \rho) \cos ^{2}(q m \phi)=\frac{1}{2 q} \sum_{l=0}^{q-1}[-J_{0}\left(2 \kappa \rho \sin \psi_{l}\right) \sin ^{2} \psi_{l}-J_{0}(2 \kappa \rho \sin \pi l / q) \sin ^{2} \pi l / q \\
&\left.+\frac{J_{1}\left(2 \kappa \rho \sin \psi_{l}\right)}{2 \kappa \rho \sin \psi_{l}}+\frac{J_{1}(2 \kappa \rho \sin \pi l / q l)}{2 \kappa \rho \sin \pi l / q}\right] .
\end{aligned}
$$

By inserting equations 52 - 56 into the expressions for $\dot{\mathcal{Q}}_{a, \rho}, \dot{\mathcal{Q}}_{a, \phi}$ and following the same procedure that led to 46), we finally get

$$
\begin{aligned}
& \dot{\mathcal{Q}}_{a, \phi}=-\frac{1}{2} \sum_{b<a} \hbar c\left|k_{a b}\right| \Gamma_{a \rightarrow b}^{\phi}\left\{\frac{2}{3}-H_{\phi}\left(2\left|k_{a b}\right| \rho, \phi\right)-\sum_{l=1}^{q-1}\left[H_{\phi}\left(2\left|k_{a b}\right| \rho, \phi+\pi l / q\right)+H_{\phi}\left(2\left|k_{a b}\right| \rho, \pi l / q\right)\right]\right\}, \\
& \dot{\mathcal{Q}}_{a, \rho}=-\frac{1}{2} \sum_{b<a} \hbar c\left|k_{a b}\right| \Gamma_{a \rightarrow b}^{\rho}\left\{\frac{2}{3}-H_{\rho}\left(2\left|k_{a b}\right| \rho, \phi\right)-\sum_{l=1}^{q-1}\left[H_{\rho}\left(2\left|k_{a b}\right| \rho, \phi+\pi l / q\right)-H_{\rho}\left(2\left|k_{a b}\right| \rho, \pi l / q\right)\right]\right\},
\end{aligned}
$$


where

$$
\begin{aligned}
H_{\phi}(x, \psi)=G_{\|}( & x \sin \psi) \sin ^{2} \psi \\
& +2 G_{\perp}(x \sin \psi) \cos ^{2} \psi \\
H_{\rho}(x, \psi)=G_{\|}( & x \sin \psi) \cos ^{2} \psi \\
& +2 G_{\perp}(x \sin \psi) \sin ^{2} \psi,
\end{aligned}
$$

and also

$$
G_{\perp}(x)=\frac{\cos x}{x^{2}}-\frac{\sin x}{x^{3}}
$$

As we now have well suited expressions for the decaying rates, we may proceed with some numerical investigations. In order to simplify our discussion, let us assume that our atom has only one dominant transition, so that we can neglect all terms in the summation of states present in 460-(58) except for this dominant term.

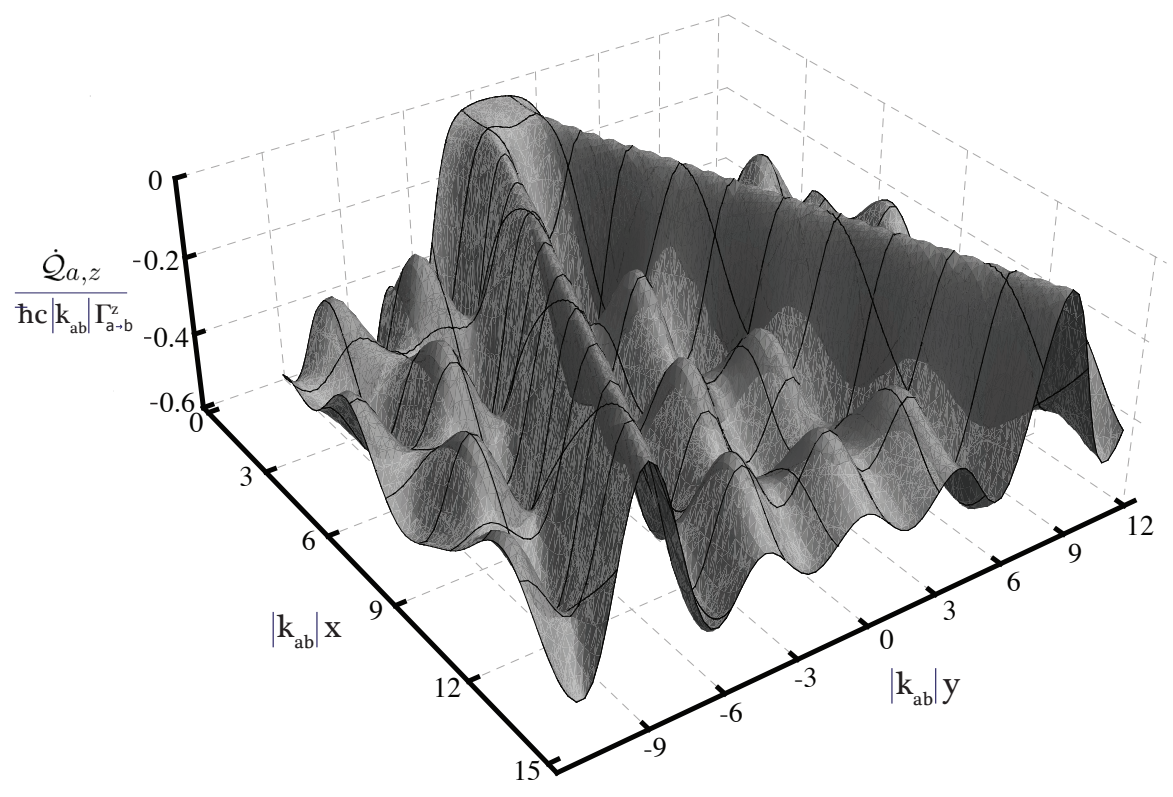

FIG. 2: The spontaneous emission for the $z$-polarization in a wedge of $\pi / 3$ radians. The axes $\mathcal{X}, \mathcal{Y}$ and $\mathcal{Z}$ follow the same conventions of figure 1 . The plates are not displayed but the plot itself is very suggestive - they would be sharply cutting the two highest slopes in half along the $\mathrm{z}$ direction. Their aspect can be easily inferred from the diffuse V-shape drawn by those slopes.

The first thing that we would like to point out is how differently each polarization responds to the presence of the wedge. By one hand, as one can see from Fig. 2. the contribution of the $z$-polarization for the spontaneous emission inside a wedge is greatly suppressed in the vicinity of the plates. However, by taking a look at the equivalent graph for the $\phi$-polarization shown in Fig. 3 , one concludes that just the opposite occurs, which means that this contribution is enhanced near the plates. This effect may seem curious initially, but it is readily understood by recalling the boundary conditions imposed by the wedge on the electric field, given by $\hat{\phi} \times\left.\mathbf{E}\right|_{S}=0$. 


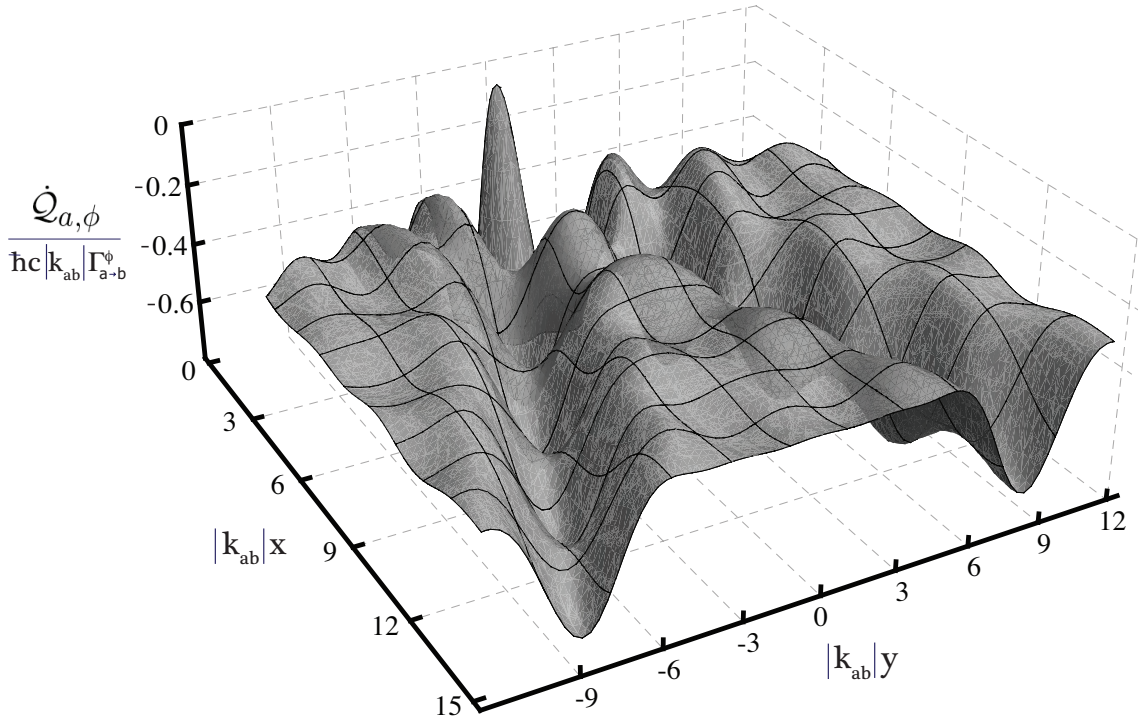

FIG. 3: The spontaneous emission for the $\phi$ polarization in the same wedge as above.

This implies that very close to one of the plates and relatively away from the cusp the electric field is approximately perpendicular to the mirror surface. Since we are working in the dipole approximation, we conclude that the parallel component of the dipole no longer couples to the field while the perpendicular component coupling is maximized. This explains both the vanishing of $\dot{\mathcal{Q}}_{a, z}$ (and also of $\dot{\mathcal{Q}}_{a, \rho}$ ) and the enhancement of $\dot{\mathcal{Q}}_{a, \phi}$ near the plates. We should mention that the previous reasoning is not valid when that atom is too close to the cusp, and we see accordingly that in such a case the SE vanishes for both polarizations. This is, of course, a direct consequence of the vanishing of the electric field at the cusp, as it may be seen from expressions (24) and (25).

Intuitively, we should expect that a wedge characterized by very small angles should mimic the behavior of two parallel plates, provided the atom is not too close to the cusp. The fact that we have checked numerically that our expressions coincide with the known results for parallel plates in the limit $\alpha \rightarrow 0, \rho \rightarrow \infty, \rho \alpha \rightarrow$ const., encouraged us to consider less extreme situations, in which $\alpha$ is small but not infinitesimal. An interesting thing happens when the setup under consideration is like the one depicted in Fig. 4 , in which an atom with $\dot{\mathcal{Q}}_{a, \phi}=0$ is placed in the $X$ axis at a given distance from the cusp $\rho=x$. If $\alpha \ll 1$ it is reasonable to approximate the wedge by two parallel plates, but the distance between these effective plates changes as we move the atom along the $X$ axis, like illustrated in Fig 5 . So, by considering an atom in different positions at the bisecting plane of an acute wedge, we are approximately also looking to the situation in which the atom is halfway between two parallel plates and these plates are put at different separations. In Fig. 6 we show the behavior of $\dot{\mathcal{Q}}_{a, \|}=\dot{\mathcal{Q}}_{a, z}+\dot{\mathcal{Q}}_{a, \rho}$ as we move the atom along the $X$ axis for different values of $q$. We see that all curves are practically zero up to approximately $k_{a b} x=q$, where they rise abruptly and then start oscillating around a decaying mean value. This is more clearly understood in terms of effective parallel mirrors, as we shall see.

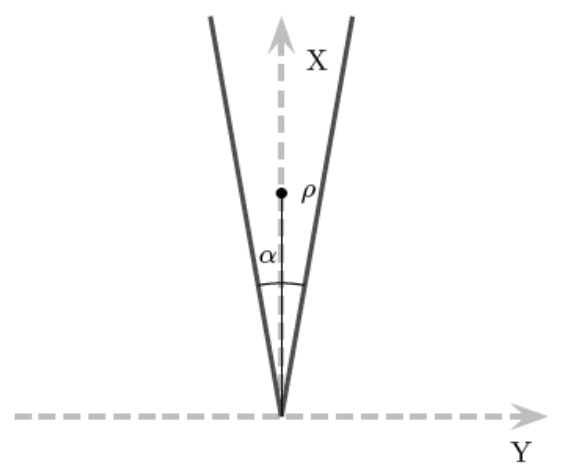

FIG. 4: An atom localized in the bisecting plane of a wedge

The perpendicular component of the wave vector characterizing the modes between two plates is discrete and given by $n \pi / \delta$, where $\delta$ is the distance between the plates. When $k_{a b} x<q$, we have necessarily $\delta<\lambda_{a b} / 2$ (reminding that $\delta \approx x \alpha=x \pi / q$ and $\left.\lambda_{a b}=2 \pi / k_{a b}\right)$. This implies that all the modes with $n>0$ are more energetic then the $a \rightarrow b$ transition, making their excitation by the dipole impossible. That said, we conclude that the only way in which the dipole could emit would be to populate the mode $n=0$, but that is also impossible since the zero mode is polarized perpendicularly to the 
plates [12, not coupling to the dipole at all [28. The situation changes dramatically once $k_{a b} x \approx q$, since the availability of at least one mixed (with perpendicular and parallel components) mode with $E_{\text {mode }}<\hbar \omega_{a b}$ allows the atom to emit, explaining the abrupt jump at that point. Based on the same reasoning, we should expect similar jumps each time the distance between the atom and the cusp reaches a multiple of $\lambda_{a b} / 2$ (or equivalently, if $k_{a b} x$ reaches a multiple of $q$ ), but as we see from the curves corresponding to $q=60$ and $q=90$, there are jumps at $k_{a b} x \approx 3 q$ but not at $k_{a b} x \approx 2 q$. This may seem strange at first sight, but it is a direct consequence of the structure of the allowed modes between two mirrors. As we said before, these modes are characterized by an integer $n$ present in the perpendicular component of the respective wave vectors. By using the explicit form of these modes [12, it is possible to show that the ones associated with even integers have a node at $\delta / 2$, producing a vanishing electric field there. Since our atom is effectively located at $\delta / 2$, we conclude that it feels only the influence of the odd modes, and that is the reason for the jumps only at $k_{a b} x$ equal to odd values of $q$.
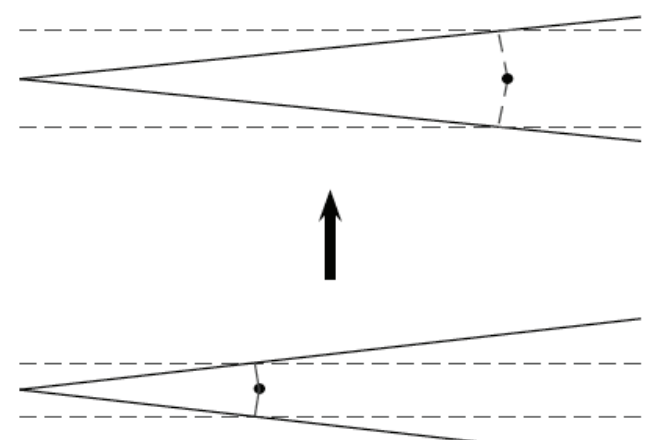

FIG. 5: Effective mirrors at two different positions of the atom. We note that the distance between the effective plates becomes larger as the atom moves away from the cusp.

We shall close this work by considering a limiting case of our expressions, in which we put $\alpha=\pi$ and the wedge degenerates into a single plate. Then $q=1$ and by eval-

Acknowledgments: FSSR and CF are very indebted to G. Barton, R. Passante and R. Rodrigues for valuable criticism and comments. FSSR would like to thank M. Moriconi for the kind hospitality at Universidade Federal Fluminense, where part of of this work was developed, uating (46, 57) and (58) at $\phi=\pi / 2$ we get

$$
\begin{gathered}
\dot{\mathcal{Q}}_{a, \|}=\dot{\mathcal{Q}}_{a, x}+\dot{\mathcal{Q}}_{a, z}=-\frac{1}{2} \hbar k_{a b} c\left(\Gamma_{a \rightarrow b}^{x}+\Gamma_{a \rightarrow b}^{z}\right) . \\
{\left[\frac{2}{3}-\frac{\sin \left(2\left|k_{a b}\right| x\right)}{2\left|k_{a b}\right| x}-\frac{\cos \left(2\left|k_{a b}\right| x\right)}{\left(2\left|k_{a b}\right| x\right)^{2}}+\frac{\sin \left(2\left|k_{a b}\right| x\right)}{\left(2\left|k_{a b}\right| x\right)^{3}}\right],} \\
\dot{\mathcal{Q}}_{a, y}=-\hbar k_{a b} c \Gamma_{a \rightarrow b}^{y}\left[\frac{1}{3}-\frac{\cos \left(2\left|k_{a b}\right| x\right)}{\left(2\left|k_{a b}\right| x\right)^{2}}\right. \\
\left.+\frac{\sin \left(2\left|k_{a b}\right| x\right)}{\left(2\left|k_{a b}\right| x\right)^{3}}\right]
\end{gathered}
$$

which is in precise agreement with the known result from the literature [4, 12].

\section{CONCLUSION AND PERSPECTIVES}

In this paper we investigated how the spontaneous emission rate of an atom is affected by the presence of a conducting wedge. We used a very general method, which allows for the evaluation of van der Waals and excited potentials as well as spontaneous emission rates. Firstly we applied it to the problem of an atom inside a mirror wedge. In the following section we showed that, although for a wedge of an arbitrary angle $\alpha$ the expressions are considerably involved, when $q \in \mathbb{N}$ a lot of simplification was possible and we arrived at manageable expressions for SE rates. We then obtained the contribution of each polarization and plotted two of them separately. Note that the behavior of the SE rate in a wedge shows the usual oscillatory pattern.

As expected, the phenomenon of suppression of the SE rate can also occur for an atom inside a wedge. As we showed graphically, for very small angles of the wedge, the plates forming it behave effectively as they were two parallel plates. Hence, for polarizations parallel to these effective plates, namely, polarizations $\rho$ and $z$, there are configurations for which the atom will not decay at all.

There are several possible generalizations for this work, the most obvious one being how to bring some real effects into consideration, like finite conductivity and temperature corrections, which fall out of the scope of this paper but serve as a guide for future research.

and also acknowledge FAPERJ for parcial financial support. Furthermore, TCNM acknowledges FAPERJ for financial support and $\mathrm{CF}$ thanks $\mathrm{CNPq}$ for partial financial support.
[1] A. Einstein, Phys. Z. 18, 121 (1917).

[2] We should mention that these are not the only novelties in this work. In this same paper Einstein discusses many other things, like the properties of the photon, which also are of key importance. For a better account of this paper accomplishments, see 3, 4. 


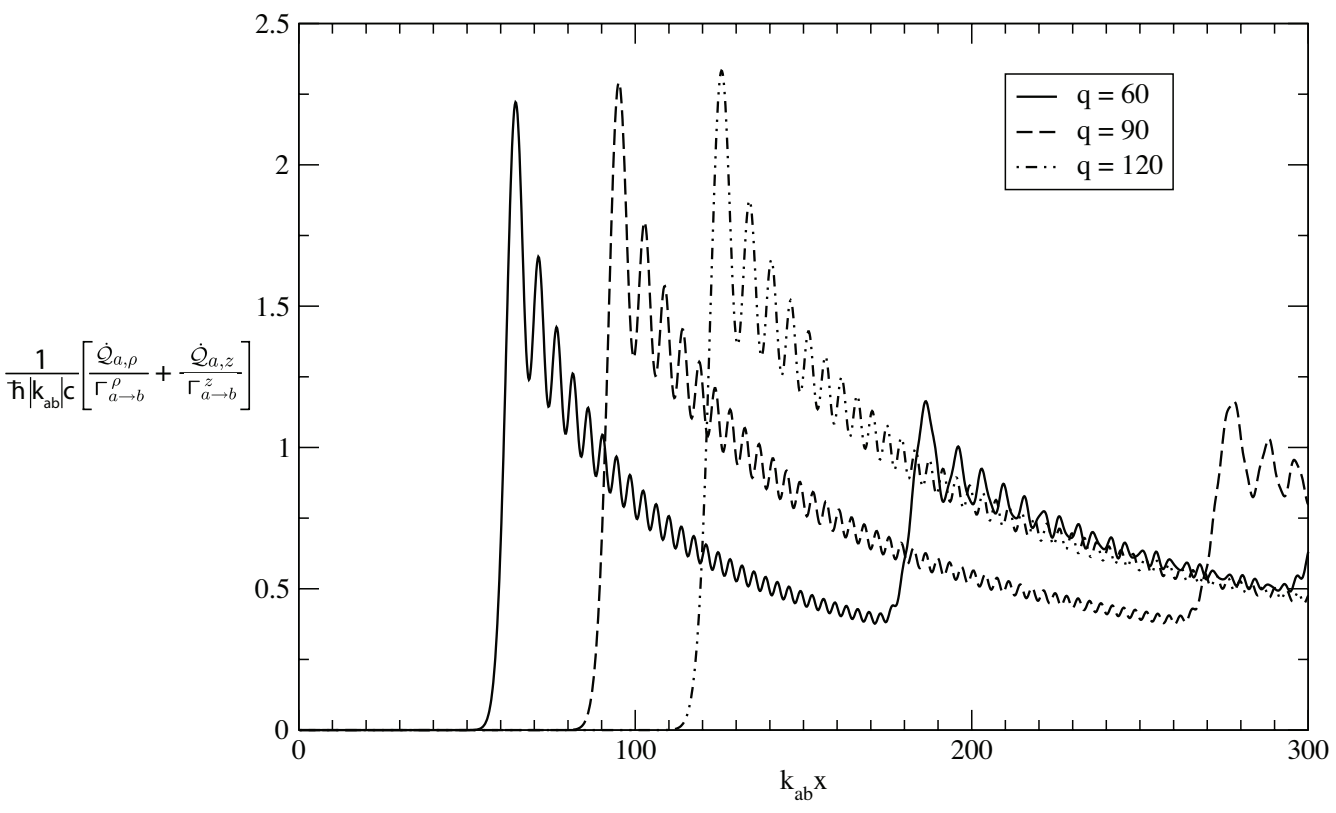

FIG. 6: Spontaneous emission for an atom polarizable only in the $\rho$ and $z$ directions and located along the bisector plane of the wedge as a function of its distance from the cusp. Note the occurrence of the phenomenon of suppression for distances from the cusp smaller than a certain value determined by the angle of the wedge, followed by a sudden jump. In the curves corresponding to $q=60$ and $q=90$ it is even possible to see further jumps (at $k_{a b} x \approx 180$ for $q=60$ and $k_{a b} x \approx 270$ for $q=90$ ), caused by the sudden availability of extra modes (see also the text).

[3] D. Klepnner, Phys. Today 2, 30 (2005).

[4] P.W. Milonni, The Quantum Vacuum, Academic Press (1994).

[5] P.A.M. Dirac, Proc. Roy. Soc. Lond. 114, 243 (1927).

[6] Actually the role of boundaries in the SE was a disputed matter sometime ago, see section 6.2 of 4 .

[7] E.M. Purcell Phys. Rev. 69, 681 (1946).

[8] G. Feher, J.P. Gordon, E. Buehler, E.A. Gere and C.D. Thurmond, Phys. Rev. 109, 221 (1958).

[9] K.H. Drexhage, H. Kuhn and F.D. Schafer, Ber. Bungen Ges. Phys. 72, 329 (1968).

[10] G. Barton, Proc. Roy. Soc. Lond. A 320, 251 (1970).

[11] P. Stehle, Phys. Rev. A 2, 95 (1970).

[12] E.A. Hinds, Advances in Atomic, Molecular and Optical Physics 28, 237 (1991).

[13] A.A. Saharian, Eur. Phys. J. C 52, 721 (2007).

[14] D. Deutsch and P. Candelas, Phys. Rev. D 20, 3063 (1979).

[15] I. Brevik and M. Lygren, Ann. Phys. (N.Y.) 251, 157 (1996)

[16] I. Brevik, M. Lygren and V.N. Marachevsky, Ann. Phys. (N.Y.) 267, 134 (1998)

[17] C.I. Sukenik, M.G. Boshier, D. Cho, V. Sandoghdar, E.A. Hinds, Phys. Rev. Lett. 70, 560 (1993).
[18] J. Dalibard, J. DuPont-Roc and C. Cohen-Tannoudji, J. Physique 43, 1617 (1982).

[19] J. Dalibard, J. DuPont-Roc and C. Cohen-Tannoudji, J. Physique 45, 637 (1984).

[20] C. Cohen-Tannoudji, J. DuPont-Roc and G. Grynberg, Atom-Photon Interactions: Basic Processes and Applications, John Wiley and Sons, New York (1992).

[21] R. Kubo, Rep. Prog. Phys. 29, 255 (1966).

[22] D. Meschede, W. Jhe and E.A. Hinds, Phys. Rev. A 41, 1587 (1990).

[23] T.N.C. Mendes and C. Farina, J. Phys A: Math. Theor. 40, 7343 (2007).

[24] T.N.C. Mendes, F.S.S. Rosa, A. Tenorio and C. Farina, J. Phys A: Math. Theor. 41, 164029 (2008).

[25] A.A. Saharian, Int. J. Mod. Phys. A 194301 (2004).

[26] P.C.W. Davies and V. Sahni, Class. Quantum Grav. 5, 1 (1988).

[27] I.S. Gradshteyn and I.M. Ryzhnik, Table of Integrals, Series and Products, 5a ed. Academic Press (1994).

[28] Incidentally, for parallel polarizations this picture also gives the right result near the cusp, where the approximation of parallel plates should no longer hold. 